\title{
FINITE ELEMENT ANALYSIS OF THE 3D THERMAL STRESS STATE IN A BRAKE DISK
}

\author{
Adam Adamowicz \\ Bialystok University of Technology (BUT), Faculty of Mechanical Engineering, Bialystok, Poland \\ e-mail: a.adamowicz@pb.edu.pl
}

\begin{abstract}
The quasi-static thermal stress state within the linear uncoupled elasticity is studied. The calculations were performed using the finite element method (MSC.Patran/MSC.Nastran). In order to examine smooth stress changes in a brake disk during braking, based on the temperature fields at particular time steps, additionally the script using Python programming language was developed. The numerical three-dimensional FE model of the brake disk for calculation of the transient temperature field was adopted from the previous author's study. A single braking process at linear deceleration and constant contact pressure was simulated. The evolutions and the contours of the components of the stress tensor as well as the equivalent Huber-Mises stress were examined. The most important aspects of the stress state during braking were discussed.
\end{abstract}

Keywords: frictional heating, temperature, thermal stresses, pad-disk brake system, finite element method

\section{Nomenclature}

$\mathbf{B}, \mathbf{D}, \mathbf{K} \quad-\quad$ matrix of differential operators, elasticity matrix and stiffness matrix

$c, h \quad-$ specific heat $[\mathrm{J} /(\mathrm{kg} \mathrm{K})]$ and heat transfer coefficient $\left[\mathrm{W} /\left(\mathrm{m}^{2} \mathrm{~K}\right)\right]$

e $\quad-$ dilatation

$E \quad-\quad$ Young's modulus [MPa]

$f \quad-$ coefficient of friction

F $\quad-\quad$ nodal force vector due to initial strain

$J_{2} \quad-$ second invariant of the deviatoric stress tensor $\left[\mathrm{MPa}^{2}\right]$

$k, K \quad-$ thermal diffusivity $\left[\mathrm{m}^{2} / \mathrm{s}\right]$ and thermal conductivity $[\mathrm{W} /(\mathrm{m} \mathrm{K})]$

$p_{0} \quad-$ contact pressure $[\mathrm{MPa}]$

$q \quad-$ heat flux density $\left[\mathrm{W} / \mathrm{m}^{2}\right]$

$r, z \quad-$ radial and axial coordinate, respectively $[\mathrm{m}]$

$r, R \quad-$ inner and outer radius, respectively [m]

$t, t_{s} \quad-$ time and braking time $[\mathrm{s}]$

$T, T_{a}, T_{0}-$ temperature, ambient temperature and initial temperature $\left[{ }^{\circ} \mathrm{C}\right]$

$\mathbf{T} \quad-$ temperature vector

$\mathbf{u} \quad-$ column vector including components of displacements of the point

$u_{r}, u_{z} \quad-$ displacement components in radial $r$ and axial $z$ direction, respectively [m]

Greek symbols
$\alpha_{T} \quad-\quad$ thermal expansion coefficient $\left[\mathrm{K}^{-1}\right]$
$\Gamma \quad-$ area of the friction surface on the disk (within the pad)
$\delta \quad-$ thickness $[\mathrm{m}]$ 


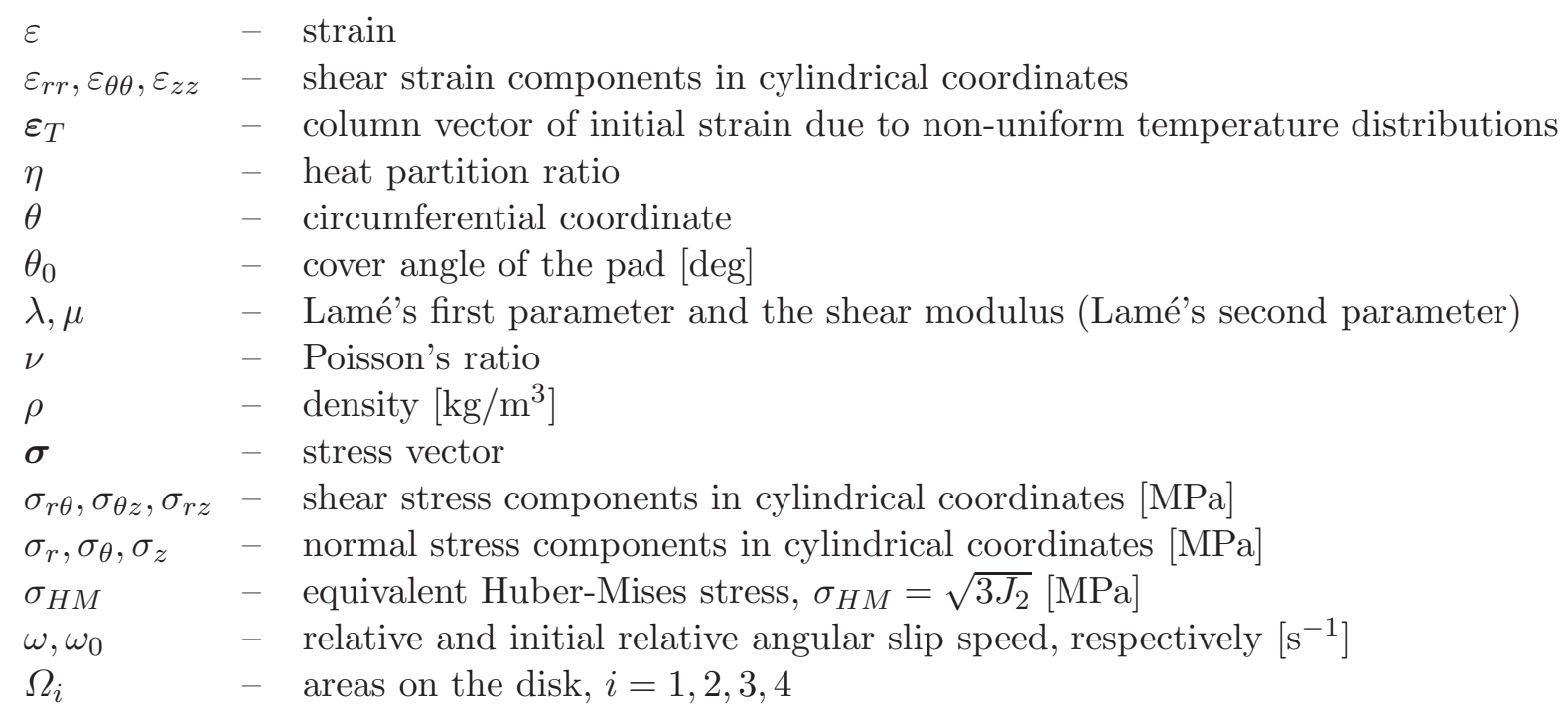

Subscripts

$d-$ disk, $p-\operatorname{pad}$

\section{Introduction}

Temperature due to friction is one of the utmost importance factors affecting performance of brake systems. A review of studies on the methods of numerical evaluation of temperature field in a pad-disk brake system encloses the work by Yevtushenko and Grzes (2010). In that paper, the drawn conclusions indicated that numerical analysis by using FEM most frequently refers to two different models. The first of them is an axisymmetric (2D) model, allowing, in particular, determination of the average temperature on the friction surface of the disk (Talati and Jalalifar, 2008; 2009; Grześ, 2010; Yevtushenko and Grzes, 2012). The advantage of using two dimensionality is the ability of immediate evaluation of the average (axisymmetric) temperature field and the corresponding thermal stresses. The drawback of the model is lack of possibility of accounting for the oscillating behavior of the contact temperature change of the brake disk during operation, which means impossibility of calculation of its maximum value. In order to determine the highest value attained, spatial FE models of the brake disk were developed (Adamowicz and Grzes, 2011a,b, 2013).

Non-uniformity of the time-spatial temperature field induces thermal stresses due to thermal expansion (Ranaker, 2001). It may, in turn, initiate micro-cracks on the friction surface of the disk, their growth and the disabling of proper and safe exploitation of a disk brake system (Mackin et al., 2002).

A review of studies on FE modeling of thermal stresses in disk brakes and clutches was given by Yevtushenko et al. (2014). It was shown that the vast majority of papers was devoted to the equivalent stress (Huber-Mises) only. However, the application of some fracture criterions enforces estimation of the evolution and spatial distribution of each component of the stress tensor in the disc brake (Norlander, 2005).

The axisymmetric quasi-static thermal stress state induced in the disk was studied using the finite element method in the first part of that research (Adamowicz, 2015). Accordingly, some papers on the development of 3D models of calculation of thermal stresses will be enclosed in this study.

Thermal fatigue fracture mechanisms of brake disks using FEM were studied by Gao et al. (2007). Temperature and thermal stresses based on the thermomechanical 3D contact model of 
a pad-disk brake system were calculated using the FE based software ANSYS 8.1. The authors discussed in detail the correlations between the obtained results and the outcomes of other studies on FE calculations of thermal stresses in disk brakes. An attempt was made to identify the cause of the thermal fatigue. Temperature distributions on the contact surface of the disk at specific time steps were shown. Radial, circumferential and equivalent Huber-Mises stresses versus braking time were presented and analyzed.

Other FE calculations for a brake disk of a high speed rail (TGV) were carried out by Tirovic (1998). Shape optimization preventing from excessive deformations was conducted. A linear elastic range of material behavior and temperature-dependent properties were imposed in the computations. A three-dimensional section $\left(7.5^{\circ}\right)$ of a ventilated type of a disk brake was analyzed. Spatial temperature, displacement, and equivalent Huber-Mises stress distributions for one specific point time were shown.

A coupled thermoelasticity problem for a pad-disk brake system during multiple braking at a constant deceleration was considered by Choi and Lee (2004). Normal stress components were determined. The influence of physical (specific heat, thermal expansion coefficient, thermal conductivity) and mechanical properties (Young's modulus) of materials on the temperature field and the real contact area were studied. It was found that the maximum value among the stress components was reached for circumferential stress which increased with temperature and the number of brake applications.

The problem of thermal cracking of cast-iron disks of trucks was studied by Bagnoli et al. (2009). Based on macro-fractography, optical and scanning electron microscopes, several radial cracks were identified. In order to eliminate one of the source of cracking originated from material defects, measurements of chemical composition, structure and hardness were made. Further numerical calculations using the finite element method also revealed a relatively high temperature and the equivalent Huber-Mises stress. It was established that the dominant factor leading to the propagation of radial macro-cracks in the heating area of semi-elliptic shape, which grew from the contact surface into the disk, was the thermal fatigue mechanism.

Thermal stresses in a ventilated brake disk induced at a uniform and non-uniform distribution of the contact pressure were analyzed by Kim et al. (2008). The contact three-dimensional computational model incorporating only mechanical interactions was developed to calculate contact pressure distributions. Further analysis of the stress field revealed that the highest values of the equivalent stress occurring for the case of the nonuniform pressure distribution was attained in the contact region. At the uniform pressure distribution, the maximum equivalent stresses were slightly higher (about 3\%) and were reached in the contact surface near the inner cylindrical surface of the disk.

Spatial FE models of three different types of ventilated and one solid disks were developed to analyze the stress field during braking (Yildiz and Duzgun, 2010). For each of the studied geometrical types of the disk, the influence of variable loading on the brake pads was also analyzed. The stresses resulted from nonuniform temperature distribution were not taken into account. In order to minimize the resulting stress, modifications of the loading of the pad were made.

Temperature distributions, equivalent Huber-Mises stresses and strains in a mine hoist brake disc were studied by Scieszka and Zolnierz (2014). The calculations were carried out both for a solid and divided disc (2, 4 and 8 sections) using a 3D thermomechanical finite element contact model. The model was validated by infrared measurements of the temperature field in real industrial conditions.

In this paper $\mathrm{FE}$ analysis of thermal stresses induced in a brake disk using fully three-dimensional model is carried out. The emphasis is placed on comprehensive examination of spatial distributions of each component of the stress tensor as well as their changes throughout the entire braking process. In order to simulate nonuniform heating of the rubbing path of the 
disk due to the fact that the cover angle of the pad is smaller than $2 \pi$, an approach to program the boundary conditions has been adopted from the previous authors' study (Yevtushenko and Grzes, 2011). Quasi-static thermal stresses are determined based on the computed transient temperature fields at the specified time moments to obtain their smooth changes during braking. The script code has been written using Python programming language and the input files to the FE based software (MSC.Nastran) have been generated. Relevant conclusions regarding thermal fatigue cracking are drawn and discussed.

\section{Statement of the problem}

A scheme of a pad-disk system given in cylindrical coordinates $r, \theta, z$ is shown in Fig. 1. All of the values and parameters, which refer to the pad and the disk in the following considerations will have subscripts $p$ and $d$, respectively. It is assumed that:

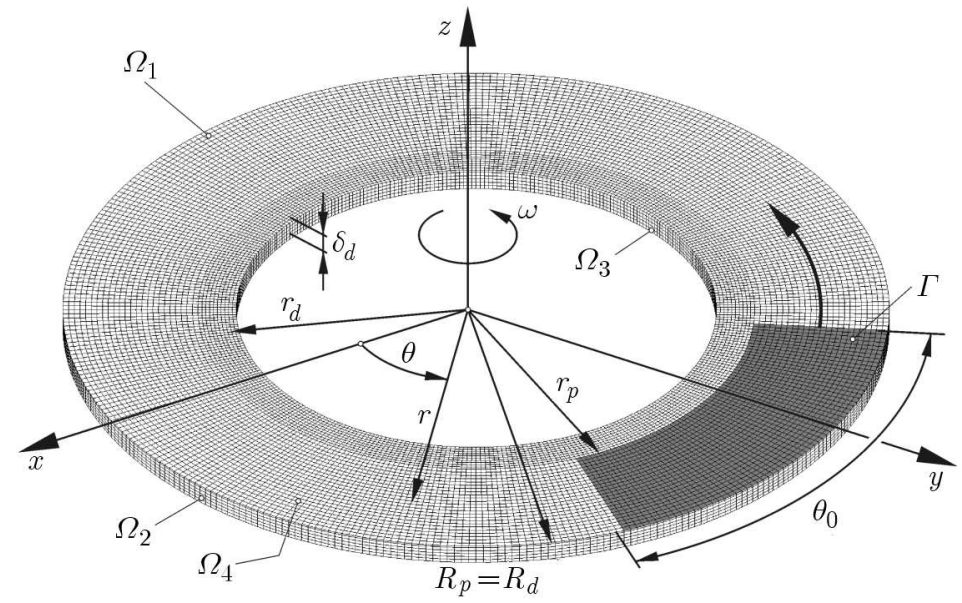

Fig. 1. A schematic diagram of a pad-disc brake system with boundary conditions and a section of the 3D FE mesh

- initially $(t=0)$, the pad is pressed to the rubbing path of the disk within the region $\Gamma=\left\{r_{p} \leqslant r \leqslant R_{p}, 0 \leqslant \theta \leqslant \theta_{0}, z=0\right\}$. The distribution of pressure in the contact area $\Gamma$ is uniform and equals $p_{0}$;

- angular speed of the disk decreases linearly from the speed of $\omega_{0}$ at the initial time moment $t=0$ to standstill at $t=t_{s}$

$$
\omega(t)=\omega_{0}\left(1-t / t_{s}\right) \quad 0 \leqslant t \leqslant t_{s}
$$

- due to friction, heat generation takes place in the contact region $\Gamma$. The heat flux densities directed along the normal into the pad and the disk (within $\Gamma$ ) are equal to $q_{p}(r, t)=$ $(1-\eta) f \omega(t) r p_{0}$ and $q_{d}(r, t)=\eta f \omega(t) r p_{0}$, respectively. Thus, the sum of these densities is equal to the specific power of friction $q(r, t)=f \omega(t) r p_{0}, r_{p} \leqslant r \leqslant R_{p}, 0 \leqslant t \leqslant t_{s}$ (Ling, 1959);

- influence of the pad on temperature of the disk is incorporated through the heat partition ratio $\eta$, while calculating this parameter in pad-disk brake systems, Charron's formula can be used (Charron, 1943)

$$
\eta=\frac{\sqrt{K_{d} \rho_{d} c_{d}}}{\sqrt{K_{d} \rho_{d} c_{d}}+\sqrt{K_{p} \rho_{p} c_{p}}}
$$


- during single braking, the heat transfer through convection and radiation on the free surfaces of the disk is negligibly small (Adamowicz and Grzes, 2011a,b). Therefore, in this study, convective cooling and thermal radiation was omitted;

- due to geometric and loading symmetry of the problem about the mid-plane of the disk, the computational region is restricted to the half $\delta_{d}$ of the disk thickness;

- materials of the pad and the disk are homogeneous and isotropic. Their physical and mechanical properties are temperature independent.

On these assumptions, the distribution of the transient 3D temperature field $T(r, \theta, z, t)$ in the disk was obtained on the basis of the FE solution to the spatial boundary heat conduction problem of the parabolic type in the paper by Adamowicz and Grzes (2013). The objective of that study was to evaluate the corresponding components of thermal stress $\sigma_{i j}, i, j=r, \theta, z$ in the disk volume from the solution to Navier's equations for uncoupled thermoelasticity given in the cylindrical coordinate system (Noda et al., 2003)

$$
\begin{aligned}
& (\lambda+2 \mu) \frac{\partial e}{\partial r}-2 \mu\left(\frac{1}{r} \frac{\partial \omega_{z}}{\partial \theta}-\frac{\partial \omega_{\theta}}{\partial z}\right)=\alpha_{T} \frac{\partial T}{\partial r} \\
& (\lambda+2 \mu) \frac{1}{r} \frac{\partial e}{\partial \theta}-2 \mu\left(\frac{\partial \omega_{r}}{\partial z}-\frac{\partial \omega_{z}}{\partial r}\right)=\alpha_{T} \frac{1}{r} \frac{\partial T}{\partial \theta} \\
& (\lambda+2 \mu) \frac{\partial e}{\partial z}-\frac{2 \mu}{r}\left(\frac{\partial\left(r \omega_{\theta}\right)}{\partial r}-\frac{\partial \omega_{r}}{\partial \theta}\right)=\alpha_{T} \frac{\partial T}{\partial z}
\end{aligned}
$$

where

$$
\begin{aligned}
e & \equiv \varepsilon_{r r}+\varepsilon_{\theta \theta}+\varepsilon_{z z}=\frac{\partial u_{r}}{\partial r}+\frac{u_{r}}{r}+\frac{1}{r} \frac{\partial u_{\theta}}{\partial \theta}+\frac{\partial u_{z}}{\partial r} \\
\omega_{r} & =\frac{1}{2}\left(\frac{1}{r} \frac{\partial u_{z}}{\partial \theta}-\frac{\partial u_{\theta}}{\partial z}\right) \quad \omega_{\theta}=\frac{1}{2}\left(\frac{\partial u_{r}}{\partial z}-\frac{\partial u_{z}}{\partial r}\right) \quad \omega_{z}=\frac{1}{2}\left(\frac{\partial\left(r u_{\theta}\right)}{\partial r}-\frac{\partial u_{r}}{\partial \theta}\right) \\
\lambda & =\frac{\nu E}{(1+\nu)(1-2 \nu)} \quad \mu=\frac{E}{2(1+\nu)} \quad \alpha_{T}=\frac{\alpha E}{1-2 \nu}
\end{aligned}
$$

A system of partial differential equations (2.3) and (2.4) will be solved for the following homogeneous (tractions free) boundary conditions (Fig. 1):

- on the surface of friction (the working surface) $\Gamma \cup \Omega_{1}=\left\{r_{d} \leqslant r \leqslant R_{d}, 0 \leqslant \theta \leqslant 2 \pi, z=0\right\}$

$$
\sigma_{z}=\sigma_{r z}=\sigma_{z \theta}=0
$$

- on the outer cylindrical surface of the disk $\Omega_{2}=\left\{r=R_{d}, 0 \leqslant \theta \leqslant 2 \pi, 0 \leqslant z \leqslant \delta_{d}\right\}$

$$
\sigma_{r}=\sigma_{r \theta}=\sigma_{r z}=0
$$

- on the inner cylindrical surface of the disk $\Omega_{3}=\left\{r=r_{d}, 0 \leqslant \theta \leqslant 2 \pi, 0 \leqslant z \leqslant \delta_{d}\right\}$

$$
\sigma_{r}=\sigma_{r \theta}=\sigma_{r z}=0
$$

- on the plane of symmetry $\Omega_{4}=\left\{r_{d} \leqslant r \leqslant R_{d}, 0 \leqslant \theta \leqslant 2 \pi, z=-\delta_{d}\right\}$

$$
u_{z}=0 \quad \sigma_{r z}=\sigma_{z \theta}=0
$$

where (Noda et al., 2003)

$$
\begin{array}{ll}
\sigma_{i i}=2 \mu \varepsilon_{i i}+\lambda e-\alpha_{T} T, \quad \sigma_{i j}=2 \mu \varepsilon_{i j} \quad i, j=r, \theta, z & i \neq j \\
\varepsilon_{r r}=\frac{\partial u_{r}}{\partial r} \quad \varepsilon_{\theta \theta}=\frac{u_{r}}{r}+\frac{1}{r} \frac{\partial u_{\theta}}{\partial \theta} \quad \varepsilon_{z z}=\frac{\partial u_{z}}{\partial z} & \\
\varepsilon_{r z}=\frac{1}{2}\left(\frac{\partial u_{r}}{\partial z}+\frac{\partial u_{z}}{\partial r}\right) \quad \quad \varepsilon_{r \theta}=\frac{1}{2}\left(\frac{1}{r} \frac{\partial u_{r}}{\partial \theta}+\frac{\partial u_{\theta}}{\partial r}+\frac{u_{\theta}}{r}\right) & \varepsilon_{z \theta}=\frac{1}{2}\left(\frac{\partial u_{\theta}}{\partial z}+\frac{1}{r} \frac{\partial u_{z}}{\partial \theta}\right)
\end{array}
$$

The solution to the boundary-value problem of quasi-static thermoelasticity, Eqs. (2.3) and (2.9)-(2.12) has been obtained using the finite element method. 


\section{FE discretization}

A general FE procedure to obtain the solution to the boundary-value heat conduction problem under consideration was presented by Adamowicz and Grzes (2013). Therefore, below we shall present in general the form of the computational scheme of the solution by means of FEM for the corresponding problem of thermoelasticity.

Taking into account formula $(2.4)_{3}$, relationships between stress and strain for Eqs. (2.9), may be written in the following matrix notation (Huebner and Thornton, 1982)

$$
\boldsymbol{\sigma}=\mathbf{D B u}-\mathbf{D} \varepsilon_{T}
$$

where

$$
\begin{array}{rlrl}
\mathbf{D} & =\frac{\lambda}{a}\left[\begin{array}{cccccc}
1 & a & a & 0 & 0 & 0 \\
a & 1 & a & 0 & 0 & 0 \\
a & a & 1 & 0 & 0 & 0 \\
0 & 0 & 0 & b & 0 & 0 \\
0 & 0 & 0 & 0 & b & 0 \\
0 & 0 & 0 & 0 & 0 & b
\end{array}\right] \quad \mathbf{B}=\left[\begin{array}{ccc}
\partial / \partial r & 0 & 0 \\
0 & \partial / \partial z & 0 \\
r^{-1} & 0 & r^{-1} \partial / \partial \theta \\
\partial / \partial z & \partial / \partial r & 0 \\
0 & r^{-1} \partial / \partial \theta & \partial / \partial z \\
r^{-1} \partial / \partial \theta & 0 & \partial / \partial r-r^{-1}
\end{array}\right] \\
\boldsymbol{\sigma} & =\left[\sigma_{r}, \sigma_{z}, \sigma_{\theta}, \sigma_{r z}, \sigma_{z \theta}, \sigma_{r \theta}\right]^{\mathrm{T}} & \mathbf{u}=\left[u_{r}, u_{z}, u_{\theta}\right]^{\mathrm{T}} & \boldsymbol{\varepsilon}_{T}=\alpha_{T} T[1,1,1,0,0,0]^{, \mathrm{T}}
\end{array}
$$

and

$$
a=\frac{\nu}{1-\nu} \quad b=\frac{1-2 \nu}{2(1-\nu)}
$$

and $T$ is the temperature increase relative to the reference temperature $T_{0}$ for which thermal strains are zero. Taking into account Eqs. (2.4) and using the variational principle of minimization of the functional of total potential energy, the boundary problem, Eqs. (2.3) and (2.5)-(2.8), leads to the system of linear algebraic equations (Zienkiewicz et al., 2005)

$$
\mathbf{K u}=\mathbf{F}
$$

where $\mathbf{K}$ is the stiffness matrix, $\mathbf{F}$ - nodal force vector due to initial strain.

Thermal FE analysis contrary to the foregoing structural analysis is a scalar field problem. The temperature field of the FE model is continuous within elements and across interelement boundaries. Temperature gradients, similarly to strains in the stress analysis, are typically not interelement-continuous (Cook, 1995). Therefore, the mesh adequate for thermal analysis might not be adequate for stress analysis unless sufficient refinement of the grid or higher order elements are imposed. In this study, to assure appropriate accuracy of the solution using the same mesh for two studied problems, a relatively fine mesh using 'CHEXA8' type eight-node finite elements has been generated. The total overall mesh consists of 86040 elements and 102960 nodes (Fig. 1).

In the preliminary analysis, two FE meshes have been tested. Robust thermal load transferred through the pad-disk interface during a short time results in a high axial component of the temperature gradient. Thus, to assure precise and reliable outcomes, a reasonably small dimension of the element in the axial direction near the friction surface (plane) ought to be used. However, an increase in the overall total number of elements of the model obviously requires larger computational resources and lengthens the time of computations. Accordingly, different meshes have been constructed and the differences between the obtained temperature changes on the contact surface during braking have been analyzed. Figure 2 shows the results determined using two grids consisting of 360 and 1080 elements in the circumferential direction. The number of the elements in the radial direction is chosen so as to maintain the square shape of an element. 
As can be seen, an increase in the number of elements does not cause significant temperature differences for the grid, therefore, its smaller number is chosen.

However, the FE based software chosen (MSC.Patran/MSC.Nastran, 2002) allowed one to conduct transient thermal analysis, it did not provide a possibility to automatically calculate stress changes based on the previously calculated temperature fields. In order to obtain stress distribution at the specified time step of the braking process, individual static stress analysis for each time step is necessary. Aiming at obtaining smooth changes of the stress fields, extremely large amount of data ought to be processed. Thus, the original code consistent with the commands used in MSC.Patran to automatize the calculations has been developed using Python programming language. The computations have been performed without the interference of MSC.Patran at the pre-processing stage. Finished input files to the FE solver (MSC.Nastran) with temperature fields at specific time steps have been generated based on the reports derived from thermal analysis.

\section{Numerical analysis}

Calculations are made for a metal-ceramic (FMC-11) pad and a cast iron (ChNMKh) disk (Chichinadze, 1967). Dimensions, properties of the components of the brake system and operating parameters of the process are listed in Table 1.

Table 1. Dimensions (Adamowicz and Grzes, 2011b), properties of materials (Yildiz and Duzgun, 2010) of the pad-disk system and operating parameters

\begin{tabular}{|l|c|c|}
\hline \multicolumn{1}{|c|}{ Parameter } & Disk (cast iron ChNMKh) & Pad (FMC-11) \\
\hline \hline inner radius, $r[\mathrm{~mm}]$ & 66 & 76.5 \\
\hline outer radius, $R[\mathrm{~mm}]$ & \multicolumn{2}{|c|}{113.5} \\
\hline thickness, $\delta[\mathrm{mm}]$ & 51 & 10 \\
\hline pad arc length, $\theta_{0}[\mathrm{deg}]$ & 7100 & 34.5 \\
\hline thermal conductivity, $K[\mathrm{~W} /(\mathrm{mK})]$ & 1.44 & 4700 \\
\hline density, $\rho\left[\mathrm{kg} / \mathrm{m}^{3}\right]$ & 99.97 & 1.46 \\
\hline thermal diffusivity, $k \cdot 10^{5}\left[\mathrm{~m}^{2} / \mathrm{s}\right]$ & 0.29 \\
\hline Young's modulus, $E[\mathrm{GPa}]$ & $1.08 \cdot 10^{-5}$ & \\
\hline Poisson's ratio, $\nu[-]$ & \multicolumn{2}{|c|}{88.464} \\
\hline thermal expansion $\operatorname{coefficient}, \alpha_{T}\left[\mathrm{~K}^{-1}\right]$ & \multicolumn{2}{|c|}{3.96} \\
\hline initial angular speed of the disk, $\omega_{0}\left[\mathrm{~s}^{-1}\right]$ & \multicolumn{2}{|c|}{0.47} \\
\hline braking time, $t_{s}[\mathrm{~s}]$ & \multicolumn{2}{|c|}{20} \\
\hline contact pressure, $p_{0}[\mathrm{MPa}]$ & 20 \\
\hline coefficient of friction, $f$ & \multicolumn{2}{|c|}{20} \\
\hline initial temperature, $T_{0}\left[{ }^{\circ} \mathrm{C}\right]$ & \multicolumn{2}{|c|}{} \\
\hline ambient temperature, $T_{a}\left[{ }^{\circ} \mathrm{C}\right]$ & \multicolumn{2}{|c|}{} \\
\hline
\end{tabular}

The FE model of the brake disk is shown in Fig. 1. The mesh shown has been generated automatically by the code developed to evaluate boundary conditions prescribed on the rubbing path of the disk. As can be seen, the established time step for FE analysis of temperatures (Figs. 2a and 3a) as well as thermal stress components (Figs. 2bcd and 3bcd) give sufficient smoothness of their changes during the entire braking process. Therefore, neither the mesh refinement nor time step reduction has been necessary.

The time profiles of temperature in Fig. $2 \mathrm{a}$ as well as the non-zero components of the stress tensor in Figs. 2b,c and Huber-Mises stress in Fig. 2d are presented for four specific radial locations on the friction surface $(z=0)$ of the disk at $\theta=0: r_{d}=66 \mathrm{~mm}$ - inner radius of 
(a)

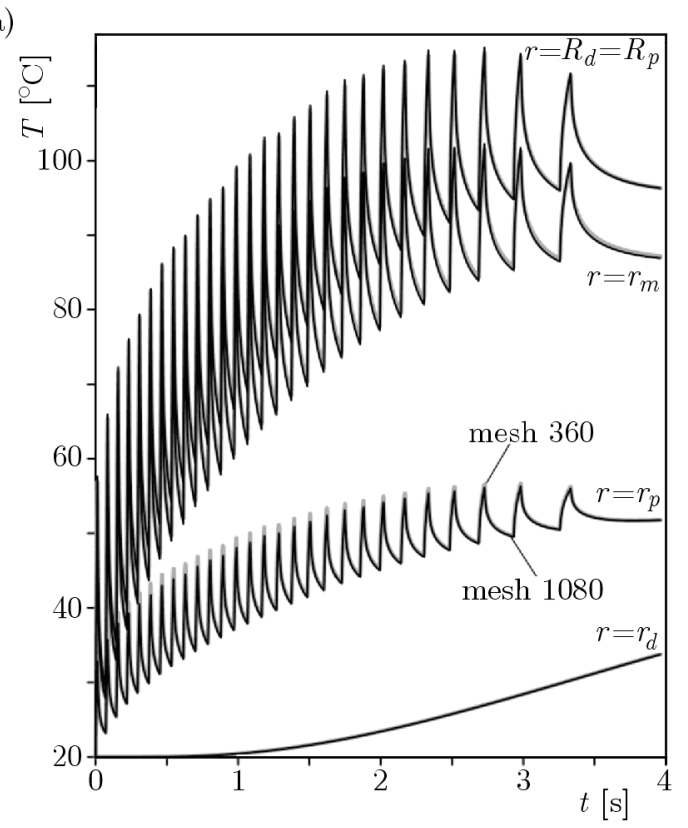

(c)

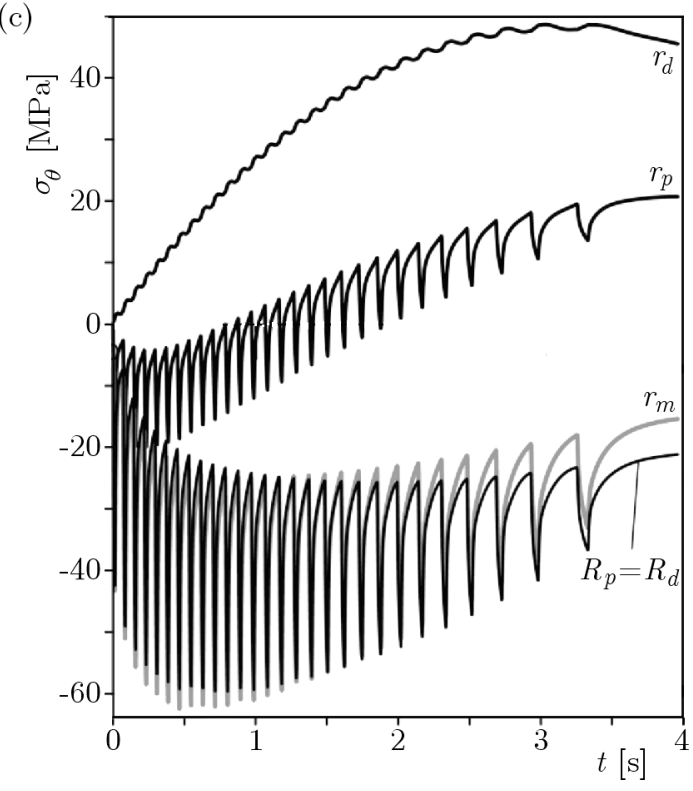

(b)

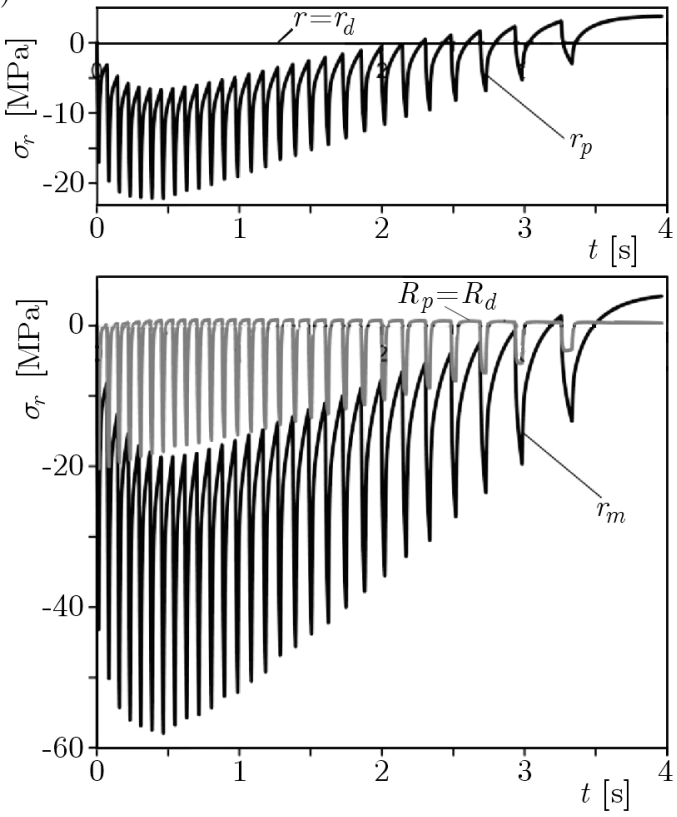

(d)

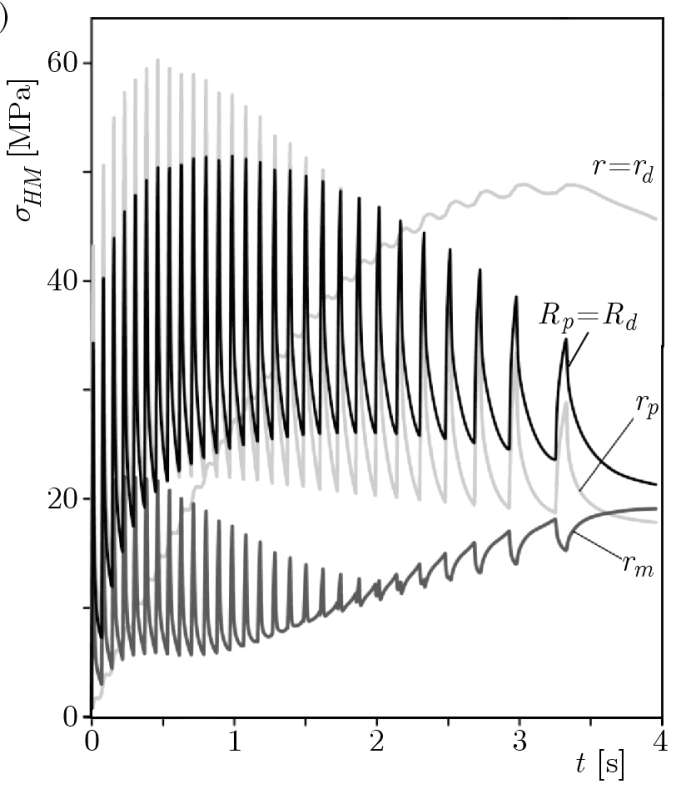

Fig. 2. Temperature (a), radial stress (b), circumferential stress (c) and equivalent Huber-Mises stress (d) changes for fixed locations on the contact surface of the disc $r \in\left\{r_{d}, r_{p}, r_{m}, R_{d}=R_{p}\right\}, \theta=0$, $z=0$ during braking

the disk, $r_{p}=76.5 \mathrm{~mm}$ - inner radius of the pad, $r_{m}=95 \mathrm{~mm}$ - mean radius of the pad, and $R_{p}=R_{d}=113.5 \mathrm{~mm}-$ outer radius of the pad and the disk.

Since the pad covers the rubbing path of the disk partly, as expected and seen in Fig. 2a, the temperature evolutions on the contact surface for fixed positions reveal fluctuations of the temperature. Only the temperature change on the inner edge of the disk varies uniformly. The average values of temperature within the rubbing path increase gradually until the maximum temperature is reached, then its slight decrease takes place. The amplitude of temperature variation during one disk revolution is attained at the beginning of the process $\Delta T=37.6^{\circ} \mathrm{C}$. The maximum temperature of the entire process occurs on the outer edge of the disk and equals $T=115.1^{\circ} \mathrm{C}(t=2.728 \mathrm{~s})$. 
The evolutions of thermal stresses corresponding with the temporal temperature profiles from Fig. 2a, are shown in Figs. 2bcd.

The radial stress $\sigma_{r}$ is negative during almost the entire period of braking, and only while approaching the stop moment, the stress changes sign to positive (Fig. 2b). The highest value $\sigma_{r}=-57.80 \mathrm{MPa}$ is reached for the mean radius $r_{m}=95 \mathrm{~mm}$ at $t=0.466 \mathrm{~s}$. According to boundary conditions (2.7), the radial stress $\sigma_{r}$ on the inner radius of the disk $(r=66 \mathrm{~mm})$ equals zero. The non-zero values of $\sigma_{r}$ at $r=113.5 \mathrm{~mm}$ stem from deformations of the outer surface of the disk.

The circumferential stress $\sigma_{\theta}$ is negative on the outer edge $(r=113.5 \mathrm{~mm})$ and inside $(r=95 \mathrm{~mm}$ ) the area of heating (Fig. 2c). While approaching the inner edge $r=76.5 \mathrm{~mm}$ of this area in a certain moment of time, there is a change in sign of this stress to positive. Note that the lack of the change in the sign in the article by Gao et al. (2007) was explained by insufficient decrease in temperature of the disk. In contrast to the radial stress, the circumferential stress reaches significant $(\approx 40 \mathrm{MPa})$ positive (tensile) values on the inner radius $r=66 \mathrm{~mm}$ of the disk. The maximum absolute values of the radial and circumferential stresses are approximately the same $(\approx 60 \mathrm{MPa})$.

(a)

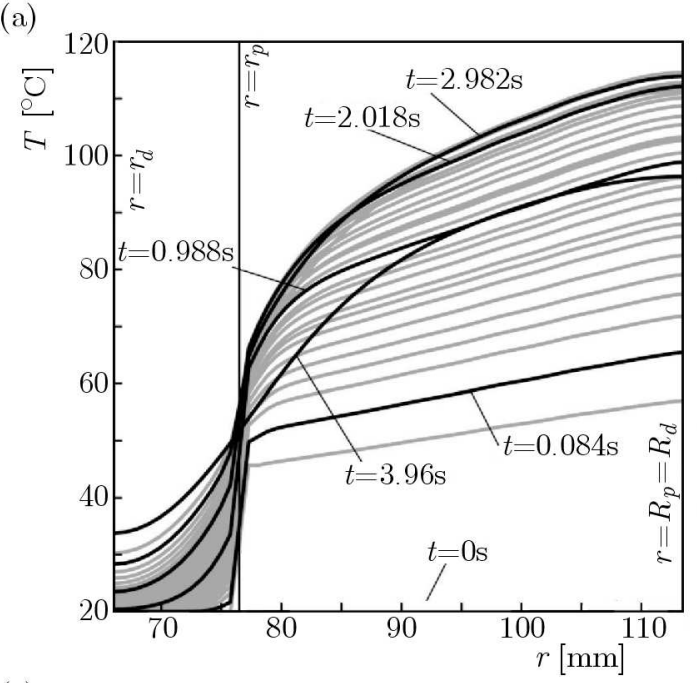

(c)

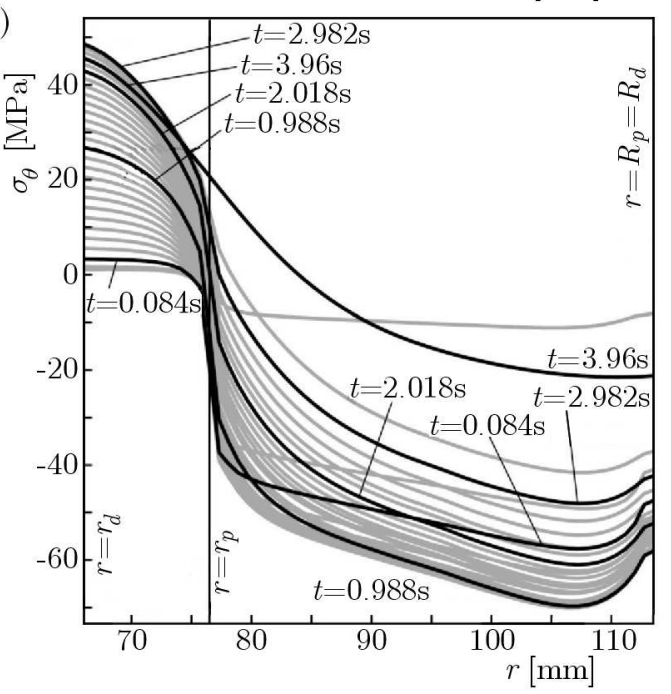

(b)

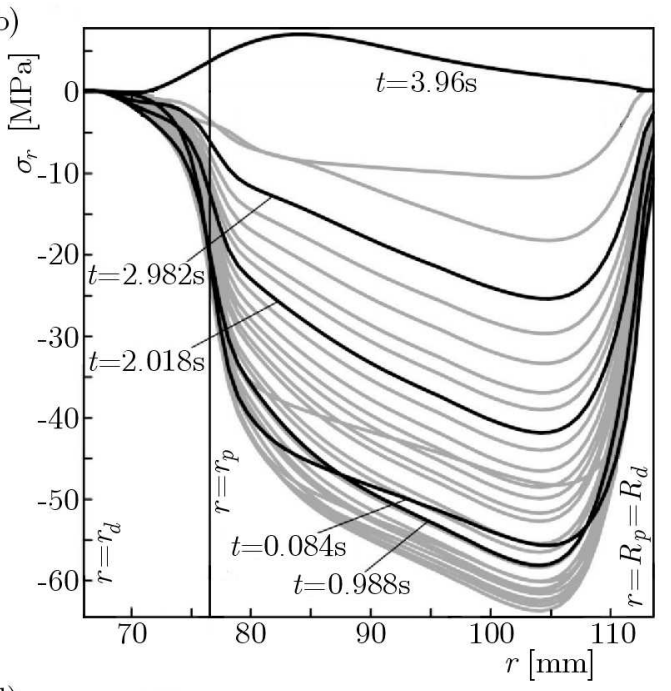

(d)

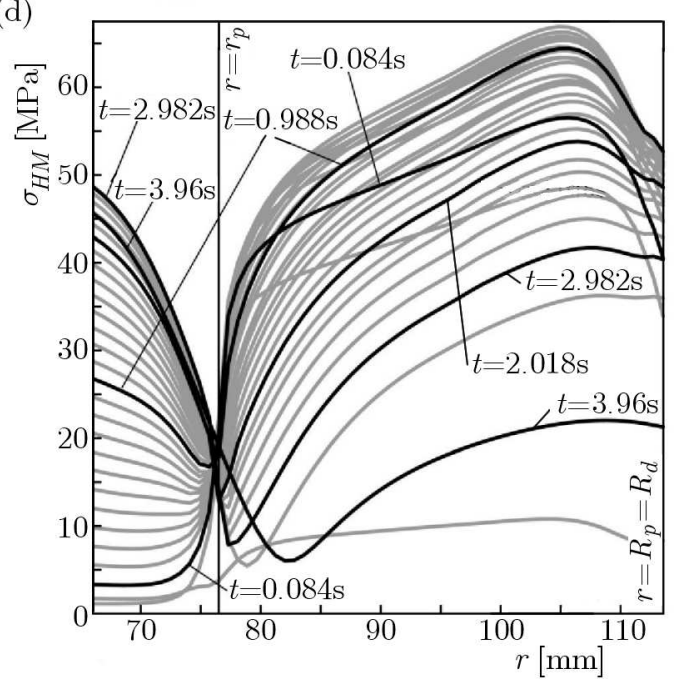

Fig. 3. Temperature (a), radial stress (b), circumferential stress (c) and equivalent Huber-Mises stress (d) distributions in the radial direction on the contact surface of disc distributions, $r_{d} \leqslant r \leqslant R_{d}$,

$$
\theta=0, z=0
$$



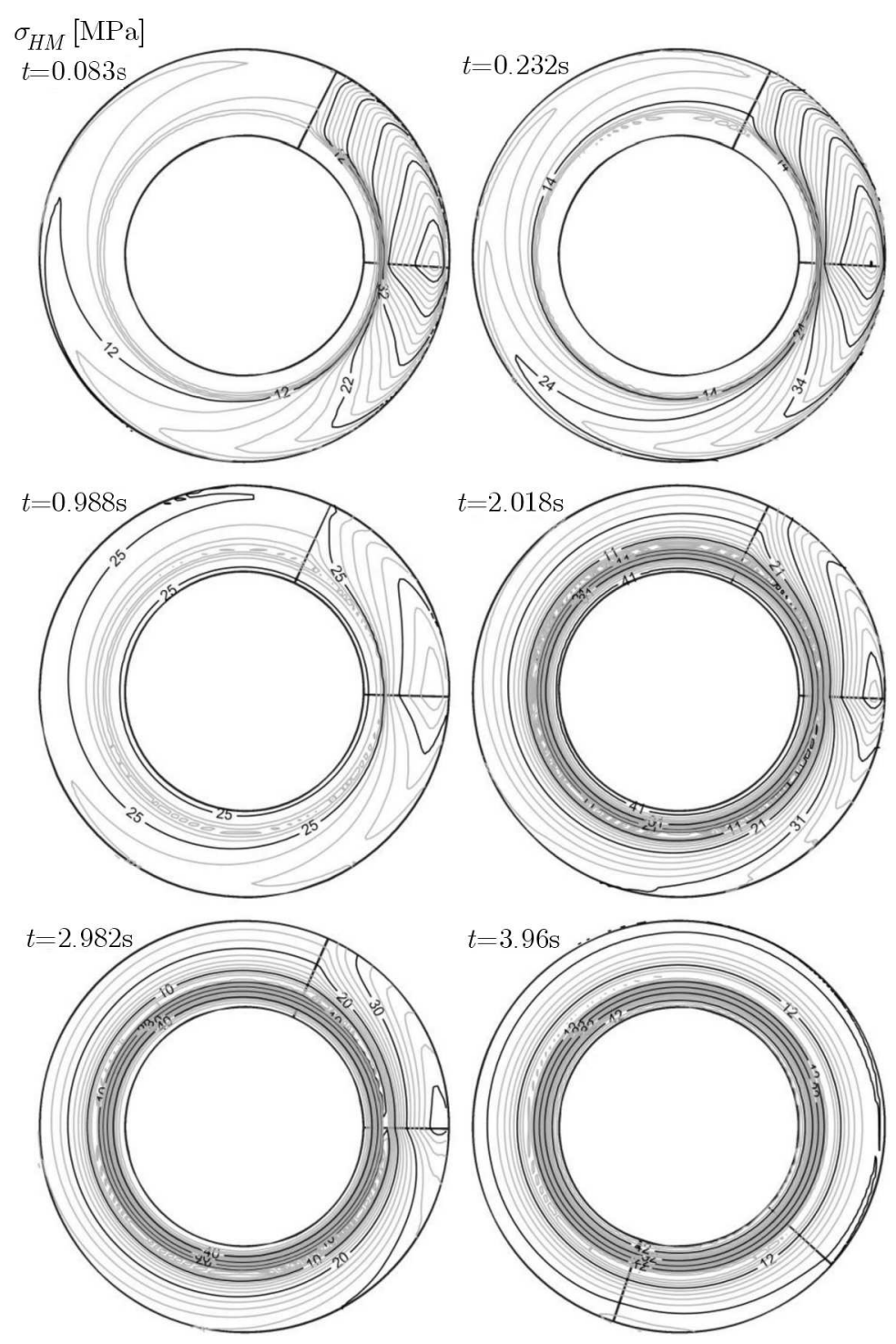

Fig. 4. Equivalent Huber-Mises stress contours on the contact surface of the disc $z=0$ at specific time moments during braking: $t=0.084 \mathrm{~s}, 0.232 \mathrm{~s}, 0.988 \mathrm{~s}, 2.018 \mathrm{~s}, 2.982 \mathrm{~s}$ and $3.96 \mathrm{~s}$

The Huber-Mises stress $\sigma_{H M}$ rises rapidly at the beginning of the process and fluctuates according to rotations of the disk/wheel (Fig. 2d). The heating (the pad covers the considered node) and cooling (the pad out of the considered node) periods may be distinguished similarly to the time profiles of temperature from Fig. 2a. Out of the rubbing path but on the friction surface $(66 \mathrm{~mm} \leqslant r \leqslant 76.5 \mathrm{~mm})$ that effect weakens. An interesting fact is that while the average values of the stress $\sigma_{H M}$ on the rubbing path decrease after reaching the maximum value at $t \approx 0.5 \mathrm{~s}$, the average values $\sigma_{H M}$ on the inner radius of the disk increase steadily almost to standstill. The maximum value $\sigma_{H M} \approx 60 \mathrm{MPa}$ on the friction surface is reached at $r=95 \mathrm{~mm}$ and the time moment $t \approx 0.5 \mathrm{~s}$. Comparable qualitative results, namely the evolutions of stresses on the friction surface and the location of the maximum value, are obtained for the thermomechanical contact FE model of the pad-disk brake system analyzed by Gao et al. (2007). However, the stress near the inner radius of the disk, unlike the considered FE model of the brake disk is relatively insignificant. This, among other things (e.g. contact pressure, properties of materials, operating parameters) may be attributed to different inner radii of the disk, since other dimensions of the pad and the disk are similar (Fig. 3a). 

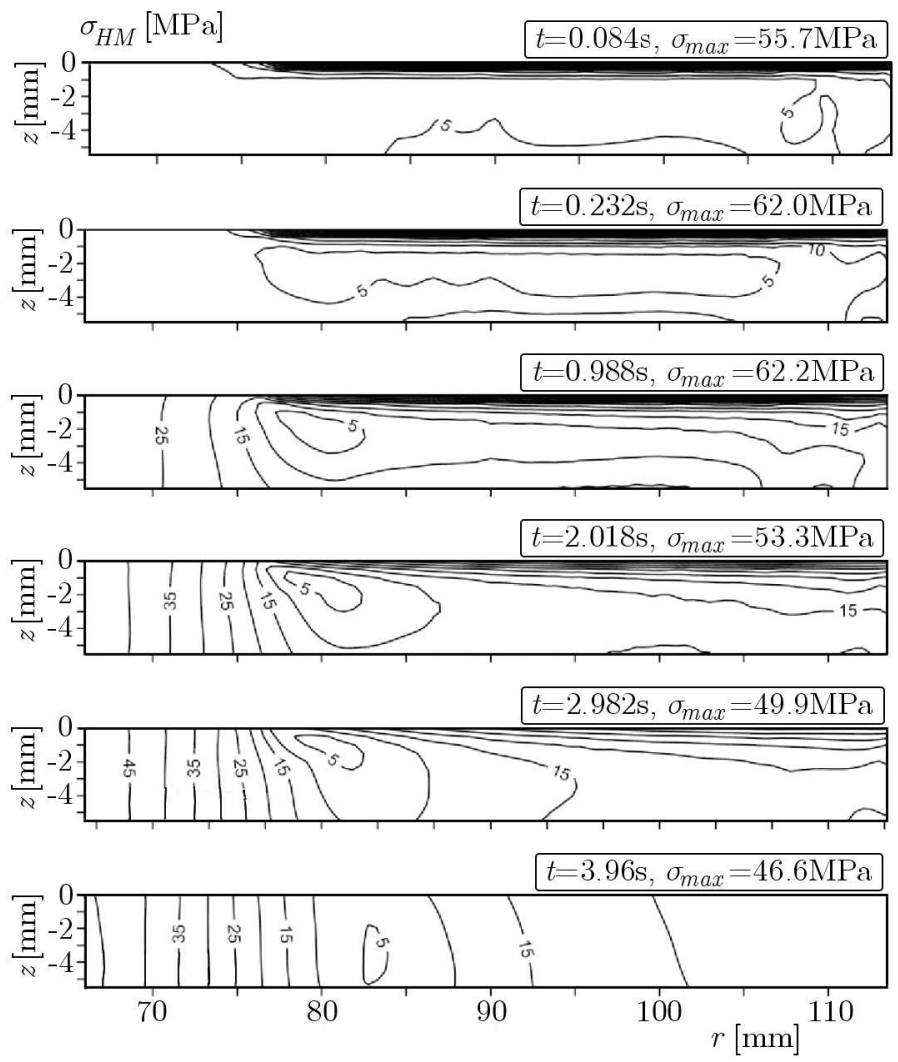

Fig. 5. Equivalent Huber-Mises stress contours in the cross-section ( $r z$ plane) of the disc at specific time moments during braking: $t=0.084 \mathrm{~s}, 0.232 \mathrm{~s}, 0.988 \mathrm{~s}, 2.018 \mathrm{~s}, 2.982 \mathrm{~s}$ and $3.96 \mathrm{~s}$

Temperature distributions and thermal stresses on the friction surface of the disk in the radial direction at selected time moments $(t=0.084 \mathrm{~s}, 0.232 \mathrm{~s}, 0.988 \mathrm{~s}, 2.018 \mathrm{~s}, 2.982 \mathrm{~s}, 3.96 \mathrm{~s})$ are shown in Fig. 3. Since the relative sliding speed of the pad and disk varies with distance from the axis of revolution $z$, the heat flux density applied on the contact surface $q=f V p$ affects the temperature distribution in the radial direction.

The distributions of stress component $\sigma_{r}$ in radial direction $r$ for the abovementioned time moments are shown in Fig. 3b. According to the imposed boundary conditions at the outer, Eq. (2.6), and inner, Eq. (2.7). cylindrical surface of the disk, the computed radial stresses should be zero. Some inconsistencies appear only on the outer edge. This is due to the software which does not use the coordinate system attached to normal directions of a deformed surface. Thus. the effect is noticeable for large stresses and deformations (from the beginning to the half of the braking process, Fig. 2b). As expected, outside these boundaries the distribution is in approximate agreement with the thermal load expressed by the product $f V p$. Additionally, it should be noted that the radial stress except the time of standstill is compressive.

In the similar way as for the radial stress distribution (within the range of the rubbing path $76.5 \mathrm{~mm} \leqslant r \leqslant 113.5 \mathrm{~mm}$ ), circumferential stresses in the radial direction reflect the influence of the thermal load on the friction surface of the disk manifested by a higher value of stress for a larger distance from the axis of revolution $\mathrm{z}$ (Fig. 3c). The circumferential stresses depicted, however, in contrast to the radial stresses (Fig. 3b) for the area free from heating on the contact surface are tensile and slightly lower than the highest compressive circumferential stresses (near the outer disk edge).

The distribution of equivalent Huber-Mises stress in the radial direction for several time moments are shown in Fig. 3d. The distribution for each time step reveals a minimum near the 
internal edge of the contact region. The location of the minimum slightly moves in the direction of the outer edge of the disk after reaching a half of the braking time.

Huber-Mises stress contours on the friction surface of the disk at certain time moments $(t=0.084 \mathrm{~s}, 0.232 \mathrm{~s}, 0.988 \mathrm{~s}, 2.018 \mathrm{~s}, 2.982 \mathrm{~s}, 3.96 \mathrm{~s})$ are depicted in Fig. 4. Concentration of the stresses follows the motion of the heating area (pad) which is particularly evident at the beginning of the process. The distribution of $\sigma_{H M}$ equalizes in the circumferential direction after about $3 \mathrm{~s}$.

Distributions of the Huber-Mises stress in the $r z$ plane at selected time moments $(t=0.084 \mathrm{~s}$, $0.232 \mathrm{~s}, 0.988 \mathrm{~s}, 2.018 \mathrm{~s}, 2.982 \mathrm{~s}, 3.96 \mathrm{~s}$ ) are shown in Fig. 5 . As can be seen, the highest values of the equivalent stress occur on the friction surface $z=0$. Accordingly, these values are consistent with the results shown in Fig. $2 d$ (the maximum stress is reached at $r=95 \mathrm{~mm}$ and $t \approx 0.5 \mathrm{~s}$. We can see also that some abrupt changes in the contour directions would suggest a slight mesh refinement in these areas.

\section{Conclusions}

The three-dimensional quasi-static thermal stresses in the brake disk using the finite element method are evaluated. Based on the obtained results general conclusions about the usefulness of the developed computational 3D FE model are drawn. This approach allows correct identification of the most strenuous disk areas. It is proved that these are: the region between the average and the outer radius of the rubbing path at the beginning of braking and the inner radius of the disk at the end of braking. Unlike the axisymmetric models, the developed spatial computational model provides valid and useful information about the stress state not only for one cycle of braking, but also distinguishes the phases of each disk rotation against the stationary pads. This may be suitable for predicting fatigue cracking processes occurring near in the zone near the contact surface of the brake disk. The periodic heating and cooling of the surface layer of the disk results in cyclic changes in the thermal stress with an amplitude of about $40 \mathrm{MPa}$, which gives the basis for the use of fatigue cracking criteria. It may be therefore established that the full thermal and mechanical stress state can only be given using a three-dimensional model.

Acknowledgement

The present paper has been financially supported by the National Science Centre in Poland (research project No. 2011/01/B/ST8/07446).

\section{References}

1. Adamowicz A., 2015, Axisymmetric FE model to analysis of thermal stresses in a brake disk, Journal of Theoretical and Applied Mechanics, 53, 2, 357-370

2. Adamowicz A., Grzes P., 2011a, Analysis of disc brake temperature distribution during single braking under non-axisymmetric load, Applied Thermal Engineering, 31, 1003-1012

3. Adamowicz A., Grzes P., 2011b, Influence of convective cooling on a disc brake temperature distribution during repetitive braking, Applied Thermal Engineering, 31, 2177-2185

4. Adamowicz A., Grzes P., 2013, Three-dimensional FE model for calculation of temperature of a thermosensitive disc, Applied Thermal Engineering, 50, 572-581

5. Bagnoli F., Dolce F., Bernabei M., 2009, Thermal fatigue cracks of fire fighting vehicles gray iron brake discs, Engineering Failure Analysis, 16, 152-163 
6. Charron F., 1943, Partage de la chaleur entre deux corps frottants, Publications Scientifiques et Techniques du Ministere de l'Air, Paris, 182

7. Chichinadze A.V., 1967, Calculation and Investigation of External Friction During Braking (in Russian), Nauka, Moscow

8. Choi J.-H., LeE I., 2004, Finite element analysis of transient thermoelastic behaviors in disk brakes, Wear, 257, 47-58

9. Cook R.D., 1995, Finite Element Modeling for Stress Analysis, John Wiley \& Sons, Inc., New York

10. Gao C.H., HuAng J.M., Lin X.Z., TAnG X.S., 2007, Stress analysis of thermal fatigue fracture of brake disks based on thermomechanical coupling, Journal of Tribology, Transactions of the ASME, 129, 536-543

11. Grześ P., 2010, Finite element analysis of temperature distribution in axisymmetric model of disc brake, Acta Mechanica et Automatica, 4, 4, 23-28

12. Huebner K.H., Thornton E.A., 1982, The Finite Element Method for Engineers, John Wiley \& Sons, New York

13. Kim D.-J., Lee Y.-M., PArK J.-S., SeOK C.-S., 2008, Thermal stress analysis for a disk brake of railway vehicles with consideration of the pressure distribution on a frictional surface, Materials Science and Engineering, A - Structural Materials Properties, 483/484, 456-459

14. LING F.F., 1959, A quasi-iterative method for computing interface temperature distribution, $Z e$ itschrift fü angewandte Mathematik und Physik (ZAMP), 10, 5, 461-475

15. Mackin T.J., Noe S.C., Ball K.J., 2002, Thermal cracking in disk brakes, Engineering Failure Analysis, 9, 63-76

16. MSC.Nastran Thermal Analysis User's Guide, 2002

17. Noda N., Hetnarski R.B., Tanigawa Y., 2003, Thermal Stresses, 2nd ed., Taylor and Francis, New York

18. Norlander M., 2005, Crack propagation in fixed caliper brake discs, Master's Dissertation, Division of Solid Mechanics, Lund University, Sweden

19. RANAKer M., 2001, Non-linear behaviour of cast iron in disc brakes - survey and calculations, Division of Machine Design, Design Sciences, LTH, Lund

20. Scieszka S.F., Zolnierz M., 2014, Experimental and numerical investigations of thermomechanical Instability of the industrial disc brakes, Proceedings of the Institution of Mechanical Engineers, Part J: Journal of Engineering Tribology, 228, 5, 567-576

21. Talati F., Jalalifar S., 2008, Investigation of heat transfer phenomena in a ventilated disk brake rotor with straight radial rounded vanes, Journal of Applied Sciences, 8, 20, 3583-3592

22. Talati F., Jalalifar S., 2009, Analysis of heat conduction in a disk brake system, Heat Mass Transfer, 45, 8, 1047-1059

23. Tirovic M., 1998, Development of a wheel mounted disc brake for a high-speed train, Proceedings of the Institution of Mechanical Engineers, Part F: Journal of Rail and Rapid Transit, 212, 2, 113-121

24. Yevtushenko A.A., Grzes P., 2010, FEM-modeling of the frictional heating phenomenon in the pad/disc tribosystem (a review), Numerical Heat Transfer, Part A: Applications, 58, 207-226

25. Yevtushenko A., Grzes P., 2011, Finite element analysis of heat partition in a pad/disc brake system, Numerical Heat Transfer, Part A: Applications, 59, 521-542

26. Yevtushenko A.A., Grzes P., 2012, Axisymmetric FEA of temperature in a pad/disc brake system at temperature-dependent coefficient of friction and wear, International Communications in Heat and Mass Transfer, 39, 8, 1045-1053 
27. Yevtushenko A.A., Grzes P., Adamowicz A., 2014, Numerical analysis of thermal stresses in disk brakes and clutches (a review), Numerical Heat Transfer, Part A: Applications, ID: 923221 DOI: $10.1080 / 10407782.2014 .923221$

28. Yildiz Y., Duzgun M., 2010, Stress analysis of ventilated brake discs using the finite element method, International Journal of Automotive Technology, 11, 133-138

29. Zienkiewicz O.C., TAylor R.L., Zhu J.Z., 2005, The Finite Element Method: its Basis and Fundamentals, Elsevier Butterworth-Heinemann, Amsterdam

Manuscript received October 17, 2014; accepted for print July 26, 2015 